Bull. Korean Math. Soc. 46 (2009), No. 2, pp. 295-301

DOI 10.4134/BKMS.2009.46.2.295

\title{
ON A STABILITY OF PEXIDERIZED EXPONENTIAL EQUATION
}

\author{
JAEyoung Chung
}

\begin{abstract}
We prove the Hyers-Ulam stability of a Pexiderized exponential equation of mappings $f, g, h: G \times S \rightarrow \mathbb{C}$, where $G$ is an abelian group and $S$ is a commutative semigroup which is divisible by 2 . As an application we obtain a stability theorem for Pexiderized exponential equation in Schwartz distributions.
\end{abstract}

\section{Introduction}

Let $f$ be a map from a vector space (or a semi group) $G$ to the field $\mathbb{C}$ of complex numbers satisfying the inequality

$$
|f(x+y)-f(x) f(y)| \leq \varepsilon \quad \text { for all } \quad x, y \in G .
$$

Then $f$ is either bounded or exponential (see [2], [3]).

When we consider the above stability problem in the spaces of generalized functions such as the Schwartz tempered distributions, Fourier hyperfunctions we encounter some stability problem of functional equation with time variables of positive real numbers while converting given distributional version of the stability problem to classical one. In this paper, we consider the stability problem of Pexiderized exponential equation with time variable

$$
|f(x+y, t+s)-g(x, t) h(y, s)| \leq \epsilon, \quad x, y \in G, t, s \in S,
$$

where $f, g, h: G \times S \rightarrow \mathbb{C}$ and $G$ is an abelian group and $S$ is a commutative semigroup divisible by 2 . As we shall see in Section 3 , the stability problem (1.2) appears when we deal with the distributional analogue of the Pexiderized version of the stability of (1.1) (see $[5,6]$ ). From now on, a function $m$ from a semigroup $\langle S,+\rangle$ to the field $\mathbb{C}$ is said to be exponential provided $m(x+y)=$ $m(x) m(y)$. As a main result we prove the following stability theorem.

Received May 15, 2008

2000 Mathematics Subject Classification. 39B82, 46F15.

Key words and phrases. distribution, Sato hyperfunction, Fourier hyperfunction, Pexiderized exponential equation, heat kernel, stability.

This work was supported by the Korean Research Foundation Grant funded by the Korean Government (MOEHRD, Basic Research Promotion Fund) (KRF-2007-521-C00016). 
Theorem 1.1. Let $f, g, h: G \times S \rightarrow \mathbb{C}$ satisfy (1.2). Then either there exist $C_{1}, C_{2}, C_{3}>0$ such that

$$
|g(x, t)| \leq C_{1}, \quad|h(x, t)| \leq C_{2}, \quad|f(x, t)| \leq C_{3}
$$

for all $(x, t) \in G \times S$, or else

$$
g(x, t)=\lambda_{1} m(t) p(x), \quad h(x, t)=\lambda_{2} m(t) p(x),\left|f(x, t)-\lambda_{1} \lambda_{2} m(t) p(x)\right| \leq \epsilon,
$$

where $\lambda_{1}, \lambda_{2} \in \mathbb{C}$ and $m: S \rightarrow \mathbb{C}$ and $p: G \rightarrow \mathbb{C}$ are exponentials.

In particular if we let $G=\{0\}$ we have $p(x) \equiv 1$ or 0 . Thus as a direct consequence of the result we have

Corollary 1.2. Let $f, g, h: S \rightarrow \mathbb{C}$ satisfy

$$
|f(t+s)-g(t) h(s)| \leq \epsilon, \quad t, s \in S .
$$

Then either there exist $C_{1}, C_{2}, C_{3}>0$ such that

$$
|g(t)| \leq C_{1}, \quad|h(t)| \leq C_{2}, \quad|f(t)| \leq C_{3}
$$

for all $t \in S$, or else

$$
g(t)=\lambda_{1} m(t), \quad h(t)=\lambda_{2} m(t),\left|f(t)-\lambda_{1} \lambda_{2} m(t)\right| \leq \epsilon,
$$

where $\lambda_{1}, \lambda_{2} \in \mathbb{C}$ and $m: S \rightarrow \mathbb{C}$ is an exponential.

Let $G=\mathbb{R}^{n}$ and $S=(0, \infty)$ and assume that $f, g, h: \mathbb{R}^{n} \times(0, \infty) \rightarrow \mathbb{C}$ are continuous functions. Then we have

Corollary 1.3. Let $f, g, h: \mathbb{R}^{n} \times(0, \infty) \rightarrow \mathbb{C}$ satisfy

$$
|f(x+y, t+s)-g(x, t) h(y, s)| \leq \epsilon, \quad x, y \in \mathbb{R}^{n}, t, s>0 .
$$

Then either there exist $C_{1}, C_{2}, C_{3}>0$ such that

$$
|g(x, t)| \leq C_{1}, \quad|h(x, t)| \leq C_{2}, \quad|f(x, t)| \leq C_{3}
$$

for all $(x, t) \in \mathbb{R}^{n} \times(0, \infty)$, or else

(1.10) $g(x, t)=\lambda_{1} e^{\lambda t+c \cdot x}, h(x, t)=\lambda_{2} e^{\lambda t+c \cdot x},\left|f(x, t)-\lambda_{1} \lambda_{2} e^{\lambda t+c \cdot x}\right| \leq \epsilon$, where $\lambda_{1}, \lambda_{2}, \lambda \in \mathbb{C}$ and $c \in \mathbb{C}^{n}$.

\section{Proofs}

Here we exclude the trivial cases $g(x, t) \equiv 0$ or $h(x, t) \equiv 0$. Replacing $(x, t)$ by $(y, s)$ and $(y, s)$ by $(x, t)$ in $(1.2)$, respectively, and using triangle inequality we have

$$
|g(x, t) h(y, s)-g(y, s) h(x, t)| \leq 2 \epsilon
$$


for all $x, y \in G, t, s \in S$. Since we exclude the trivial cases where $g(x, t) \equiv 0$ or $h(x, t) \equiv 0$, it follows from the inequality (2.1) that there exist constants $c_{1}, c_{2}, d_{1}, d_{2} \geq 0$ such that

$$
\begin{aligned}
& |g(x, t)| \leq c_{1}|h(x, t)|+d_{1}, \\
& |h(x, t)| \leq c_{2}|g(x, t)|+d_{2}
\end{aligned}
$$

for all $x \in G, t \in S$. It follows from (2.2) and (2.3) that $g(x, t)$ is bounded if and only if $h(x, t)$ is bounded. Assume that $h(x, t)$ is bounded. Putting $y=0, s=t$ in (2.1) we get (1.3). Now if $h(x, t)$ is unbounded, then we can choose $\left(y_{n}, s_{n}\right) \in \mathbb{R}^{n} \times S$ so that $\left|h\left(y_{n}, s_{n}\right)\right| \rightarrow \infty$ as $n \rightarrow \infty$. Putting $y=y_{n}, s=s_{n}$ in (1.2), dividing by $\left|h\left(y_{n}, s_{n}\right)\right|$ and letting $n \rightarrow \infty$, we have

$$
g(x, t)=\lim _{n \rightarrow \infty} \frac{f\left(x+y_{n}, t+s_{n}\right)}{h\left(y_{n}, s_{n}\right)} .
$$

Thus it follows from (1.2) and (2.4) that

$$
\begin{aligned}
g(x+y, t+s) g(0, r) & =\lim _{n \rightarrow \infty} \frac{f\left(x+y+y_{n}, t+s+s_{n}\right) g(0, r)}{h\left(y_{n}, s_{n}\right)} \\
& =\lim _{n \rightarrow \infty} \frac{g(x, t) h\left(y+y_{n}, s+s_{n}\right) g(0, r)+R_{1}}{h\left(y_{n}, s_{n}\right)} \\
& =\lim _{n \rightarrow \infty} \frac{g(x, t) f\left(y+y_{n}, s+r+s_{n}\right)+R_{1}+R_{2}}{h\left(y_{n}, s_{n}\right)} \\
& =g(x, t) g(y, s+r)+\lim _{n \rightarrow \infty} \frac{R_{1}+R_{2}}{h\left(y_{n}, s_{n}\right)},
\end{aligned}
$$

where $\left|R_{1}\right| \leq \epsilon|g(0, r)|,\left|R_{2}\right| \leq \epsilon|g(x, t)|$, which implies

$$
g(x+y, t+s) g(0, r)=g(x, t) g(y, s+r)
$$

for all $x, y \in G$ and $t, s, r \in S$. Putting $x=y=0, r=r_{0}$ in (2.5) and multiplying $g\left(0, r_{0}\right)$ in the result we have

$$
\begin{aligned}
g(0, t+s) g\left(0, r_{0}\right)^{2} & =g(0, t) g\left(0, s+r_{0}\right) g\left(0, r_{0}\right) \\
& =g(0, t) g(0, s) g\left(0,2 r_{0}\right),
\end{aligned}
$$

which implies

$$
g(0, t)=\lambda_{1} m_{1}(t)
$$

for some $0 \neq \lambda_{1} \in \mathbb{C}$ and some exponential $m_{1}$.

On the other hand, putting $x=s=0$ in (2.5) we have

$$
g(y, t) g(0, r)=g(0, t) g(y, r) .
$$

If $g(0, r)=0$ for all $r \in S$, it follows from (2.5) that $g \equiv 0$. Thus there exists $r_{0} \in S$ such that $g\left(0, r_{0}\right) \neq 0$ and it follow from (2.7) that

$$
g(y, t)=g(0, t) p(y) .
$$


Putting (2.8) in (2.5) we have

$$
p(x+y)=p(x) p(y)
$$

for all $x, y \in G$. Thus it follows from (2.6) and (2.9) that

$$
g(x, t)=g(0, t) p(x)=\lambda_{1} m_{1}(t) p(x) .
$$

Changing the roles of $g$ and $h$ we have

$$
h(x, t)=h(0, t) q(x)=\lambda_{2} m_{2}(t) q(x),
$$

where $0 \neq \lambda_{2} \in \mathbb{C}$, and $m_{2}, q$ are exponentials on $S$ and $G$, respectively.

Putting (2.10) and (2.11) in (2.1) we have

$$
\left|m_{1}(t) p(x)-\lambda m_{2}(t) q(x)\right| \leq M, \quad x \in G, t \in S
$$

for some $\lambda \in \mathbb{C}$ and $M>0$. Thus it follows from (2.12) that for each $y \in$ $G, s \in S$

$$
\begin{aligned}
& \left|m_{1}(t) p(x)\left[m_{1}(s) p(y)-m_{2}(s) q(y)\right]\right| \\
\leq & \left|m_{1}(t+s) p(x+y)-\lambda m_{2}(t+s) q(x+y)\right| \\
& +\left|m_{2}(s) q(y)\left[m_{1}(t) p(x)-\lambda m_{2}(t) q(x)\right]\right| \\
\leq & M\left(1+\left|m_{2}(s) q(y)\right|\right)
\end{aligned}
$$

for all $x \in G, t \in S$. Since $m_{1}(t) p(x)$ is unbounded we have $m_{1}(s) p(y)=$ $m_{2}(s) q(y)$ for all $y \in G, s \in S$. Finally, putting (2.10) and (2.11) in (1.2) we have the inequality

$$
\left|f(x, t)-\lambda_{1} \lambda_{2} m(t) p(x)\right| \leq \epsilon
$$

for all $(x, t) \in G \times S$. This completes the proof.

\section{Applications}

As an application we consider a distributional version of the following HyersUlam stability problem of the Pexiderized exponential equation

$$
u \circ A-v \otimes w \in L^{\infty}\left(\mathbb{R}^{2 n}\right)
$$

for $u, v, w$ in the space $\mathcal{S}^{\prime}\left(\mathbb{R}^{n}\right)$ of Schwartz tempered distributions, the space $\mathcal{F}^{\prime}\left(\mathbb{R}^{n}\right)$ of Fourier hyperfunctions and the space $\mathcal{S}_{1 / 2}^{\prime 1 / 2}\left(\mathbb{R}^{n}\right)$ of Gelfand generalized functions, where $A(x, y)=x+y, x, y \in \mathbb{R}^{n}$ and $\otimes$ denotes the tensor product of generalized functions and $L^{\infty}\left(\mathbb{R}^{n}\right)$ denotes the space of bounded measurable functions on $\mathbb{R}^{n}$.

For the space of Schwartz tempered distributions we refer the reader to $[4,8,9,13]$. Here we briefly introduce the spaces of Gelfand generalized functions and Fourier hyperfunctions. Here we use the following notations: $|x|=\sqrt{x_{1}^{2}+\cdots+x_{n}^{2}}, \quad|\alpha|=\alpha_{1}+\cdots+\alpha_{n}, \alpha !=\alpha_{1} ! \cdots \alpha_{n} !, x^{\alpha}=x_{1}^{\alpha_{1}} \cdots x_{n}^{\alpha_{n}}$ and $\partial^{\alpha}=\partial_{1}^{\alpha_{1}} \ldots \partial_{n}^{\alpha_{n}}$ for $x=\left(x_{1}, \ldots, x_{n}\right) \in \mathbb{R}^{n}, \alpha=\left(\alpha_{1}, \ldots, \alpha_{n}\right) \in \mathbb{N}_{0}^{n}$, where $\mathbb{N}_{0}$ is the set of non-negative integers and $\partial_{j}=\frac{\partial}{\partial x_{j}}$. 
Definition $3.1([8])$. For given $r, s \geq 0$ we denote by $\mathcal{S}_{r}^{s}$ or $\mathcal{S}_{r}^{s}\left(\mathbb{R}^{n}\right)$ the space of all infinitely differentiable functions $\varphi(x)$ on $\mathbb{R}^{n}$ such that there exist positive constants $h$ and $k$ satisfying

$$
\|\varphi\|_{h, k}:=\sup _{x \in \mathbb{R}^{n}, \alpha, \beta \in \mathbb{N}_{0}^{n}} \frac{\left|x^{\alpha} \partial^{\beta} \varphi(x)\right|}{h^{|\alpha|} k^{|\beta|} \alpha !^{r} \beta !^{s}}<\infty .
$$

The topology on the space $\mathcal{S}_{r}^{s}$ is defined by the seminorms $\|\cdot\|_{h, k}$ given by (3.2) and the elements of the dual space $\mathcal{S}^{\prime s}$ of $\mathcal{S}_{r}^{s}$ are called Gelfand-Shilov generalized functions. In particular, we denote $\mathcal{S}^{\prime}{ }_{1}$ by $\mathcal{F}^{\prime}$ and call its elements Fourier hyperfunctions.

It is known that if $r>0$ and $0 \leq s<1$, the space $\mathcal{S}_{r}^{s}\left(\mathbb{R}^{n}\right)$ consists of all infinitely differentiable functions $\varphi(x)$ on $\mathbb{R}^{n}$ that can be extended to an entire function on $\mathbb{C}^{n}$ satisfying

$$
|\varphi(x+i y)| \leq C \exp \left(-a|x|^{1 / r}+b|y|^{1 /(1-s)}\right)
$$

for some $a, b>0$. It is well known that the following topological inclusions hold:

$$
\mathcal{S}_{1 / 2}^{1 / 2} \hookrightarrow \mathcal{F} \hookrightarrow \mathcal{S}, \quad \mathcal{S}^{\prime} \hookrightarrow \mathcal{F}^{\prime} \hookrightarrow \mathcal{S}_{1 / 2}^{\prime 1 / 2}
$$

We denote by $E_{t}(x)$ the $n$-dimensional heat kernel

$$
E_{t}(x)=(4 \pi t)^{-n / 2} \exp \left(-|x|^{2} / 4 t\right), \quad t>0 .
$$

Let $u \in \mathcal{S}_{1 / 2}^{\prime 1 / 2}$. Then its Gauss transform $f(x, t):=\left(u * E_{t}\right)(x)=\left\langle u_{y}, E_{t}(x-\right.$ $y)\rangle$ is a $C^{\infty}$-solution of the heat equation

$$
\left(\Delta-\partial / \partial_{t}\right) f(x, t)=0
$$

in $\left\{(x, t): x \in \mathbb{R}^{n}, t>0\right\}$. Also $\left(u * E_{t}\right)(x) \rightarrow u$ as $t \rightarrow 0^{+}$in the sense of generalized functions.

Lemma 3.2 ([14]). Let $f(x, t)$ be a solution of the heat equation satisfying

$$
|f(x, t)| \leq M, \quad x \in \mathbb{R}^{n}, t \in(0,1) .
$$

Then $f$ can be written as

$$
f(x, t)=\left(f_{0} * E_{t}\right)(x)=\int f_{0}(y) E_{t}(x-y) d y
$$

for some bounded measurable function $f_{0}$ defined in $\mathbb{R}^{n}$.

Theorem 3.3. Let $u, v, w \in \mathcal{S}_{1 / 2}^{\prime 1 / 2}$ satisfy (3.1). Then $u, w, w$ are bounded measurable functions, or else

$$
v=\lambda_{1} e^{c \cdot x}, w=\lambda_{2} e^{c \cdot x}, u=\lambda_{1} \lambda_{2} e^{c \cdot x}+r(x),
$$

where $\lambda_{1}, \lambda_{2} \in \mathbb{C}$ and $c \in \mathbb{C}^{n}$ and $r$ is a bounded measurable function with $\|r\|_{L^{\infty}} \leq \epsilon$. 
Proof. Convolving with $E_{s}(x) E_{t}(y)$ in (3.1), in view of the semigroup property $\left(E_{s} * E_{t}\right)(x)=E_{s+t}(x)$ of the heat kernel, we have for some $M>0$,

$$
|f(x+y, t+s)-g(x, t) h(y, s)| \leq \epsilon
$$

for all $x, y \in \mathbb{R}^{n}, s, t>0$, where $f(x, t), g(x, t)$ and $h(x, t)$ are the Gauss transforms of $u, v$ and $w$, respectively. Now if $f(x, t), g(x, t)$ and $h(x, t)$ satisfy (1.9) in the Corollary 1.3, letting $t \rightarrow 0^{+}$we obtain $u, v$ and $w$ are bounded measurable functions by Lemma 3.2. If $f(x, t), g(x, t)$ and $h(x, t)$ satisfy (1.10), letting $t \rightarrow 0^{+}$, it is easy to see that $v=\lambda_{1} e^{c \cdot x}, w=\lambda_{2} e^{c \cdot x}$. Finally, since

$$
R(x, t):=f(x, t)-\lambda_{1} \lambda_{2} e^{\lambda t+c \cdot x}
$$

is a solution of the heat equation, applying Lemma 3.2 and letting $t \rightarrow 0^{+}$in (3.6), we have $u=\lambda_{1} \lambda_{2} e^{c \cdot x}+r$. This completes the proof.

Note that $e^{c \cdot x} \in \mathcal{F}^{\prime}$ only when $c=i a$ for some $a \in \mathbb{R}^{n}$. Thus we have the following.

Corollary 3.4. Let $u, v, w \in \mathcal{S}^{\prime}$ or $\mathcal{F}^{\prime}$ satisfy (3.1). Then $u, v$ and $w$ are all bounded measurable functions.

\section{References}

[1] J. Aczél and J. Dhombres, Functional Equations in Several Variables, Encyclopedia of Mathematics and its Applications, 31. Cambridge University Press, Cambridge, 1989.

[2] J. A. Baker, The stability of the cosine equation, Proc. Amer. Math. Soc. 80 (1980), no. $3,411-416$.

[3] J. A. Baker, J. Lawrence, and F. Zorzitto, The stability of the equation $f(x+y)=$ $f(x) f(y)$, Proc. Amer. Math. Soc. 74 (1979), no. 2, 242-246.

[4] J. Chang and J. Chung, The stability of the sine and cosine functional equations in Schwartz distributions, Bull. Korean Math. Soc. 46 (2009), no. 1, 87-97.

[5] J. Chung, Hyers-Ulam stability theorems for Pexider equations in the space of Schwartz distributions, Arch. Math. (Basel) 84 (2005), no. 6, 527-537.

[6] A A distributional version of functional equations and their stabilities, Nonlinear Anal. 62 (2005), no. 6, 1037-1051.

[7] I. Fenyö, Über eine Lösungsmethode gewisser Funktionalgleichungen, Acta Math. Acad. Sci. Hungar. 7 (1956), 383-396.

[8] I. M. Gelfand and G. E. Shilov, Generalized Functions. Vol. 2, Academic Press [Harcourt Brace Jovanovich, Publishers], New York-London, 1968.

[9] L. Hörmander, The Analysis of Linear Partial Differential Operators. I, Grundlehren der Mathematischen Wissenschaften [Fundamental Principles of Mathematical Sciences], 256. Springer-Verlag, Berlin, 1983.

[10] D. H. Hyers, G. Isac, and Th. M. Rassias, Stability of Functional Equations in Several Variables, Birkhauser Boston, Inc., Boston, MA, 1998.

[11] S.-M. Jung, Hyers-Ulam-Rassias Stability of Functional Equations in Mathematical Analysis, Hadronic Press, Inc., Palm Harbor, FL, 2001.

[12] Th. M. Rassias, Stability of mappings of Hyers-Ulam type, Hadronic Press Collection of Original Articles, 111-116. Hadronic Press, Inc., Palm Harbor, FL, 1994.

[13] L. Schwartz, Théorie des distributions, Hermann, Paris, 1966.

[14] D. V. Widder, The Heat Equation, Academic Press, New York, 1975. 
Department of Mathematics

Kunsan National University

Kunsan 573-701, KoreA

E-mail address: jychung@kunsan.ac.kr 